\title{
Development and field deployment of a mid-infrared methane sensor without pressure control using interband cascade laser absorption spectroscopy
}

\section{Chuantao Zheng, ${ }^{1,3}$ Weilin Ye, ${ }^{2,3^{*}}$ Nancy P. Sanchez, ${ }^{4}$ Chunguang $\mathrm{Li}^{1,3}$ Lei Dong, ${ }^{3,5}$ Yiding}

Wang, ${ }^{1}$ Robert J. Griffin, ${ }^{4}$ and Frank K. Tittel ${ }^{3}$

${ }^{1}$ State Key Laboratory on Integrated Optoelectronics, College of Electronic Science and Engineering, Jilin University, 2699 Qianjin Street, Changchun 130012, China

${ }^{2}$ College of Engineering, Shantou University, 243 Daxue Road, Shantou 515063, China

${ }^{3}$ Department of Electrical and Computer Engineering, Rice University, 6100 Main Street, Houston, TX 77005, USA

${ }^{4}$ Department of Civil and Environmental Engineering, Rice University, 6100 Main Street, Houston, TX 77005, USA

${ }^{5}$ State Key Laboratory of Quantum Optics and Quantum Optics Devices, Institute of Laser Spectroscopy, Shanxi University, Taiyuan 030006, China

*Corresponding author.wy10@rice.edu (Weilin Ye) 


\begin{abstract}
A mid-infrared methane $\left(\mathrm{CH}_{4}\right)$ sensor without pressure control was developed using a continuous-wave (CW) interband cascade laser (ICL) for targeting a $\mathrm{CH}_{4}$ absorption line located at $3038.5 \mathrm{~cm}^{-1}$. A multi-pass gas cell with an absorption path length of $54.6 \mathrm{~m}$ was utilized for enhancing gas absorption. The pressure inside the MPGC was measured using direct Lorentzian absorption fitting for the compensation of $\mathrm{CH}_{4}$ concentration changes resulting from pressure variations. Laboratory pressure calibration was conducted in the range of $25-800$ Torr using 1.3-, 1.5-, 1.7- and 2.1-ppmv $\mathrm{CH}_{4}$ samples. A pressure precision of $\sim 1.65$ Torr with $\mathrm{a} \sim 2.5$-s averaging time was achieved based on the measurement of a 2.1-ppmv $\mathrm{CH}_{4}$ sample at 700-Torr. Concentration level measurements of a 2.1-ppmv $\mathrm{CH}_{4}$ sample at a 700-Torr pressure yielded an Allan deviation of $2.25 \mathrm{ppbv}$ for an averaging time of $2.5 \mathrm{~s}$. The sensor functioned normally with $\mathrm{CH}_{4}$ samples at 1.0, 1.2, 1.4, 1.6 and 2.1 ppmv concentration levels as the pressure changes from 25 to 800 Torr. Indoor/outdoor $\mathrm{CH}_{4}$ concentration measurements on the Rice University campus and a field campaign in the Greater Houston Area (GHA) were conducted to evaluate the performance of the sensor system.
\end{abstract}

Keywords: Infrared absorption; Laser spectroscopy; $\mathrm{CH}_{4}$ sensor; Interband cascade laser 


\section{Introduction}

Methane $\left(\mathrm{CH}_{4}\right)$ with an atmospheric concentration level of $\sim 1.8$ parts per million by volume (ppmv) in the atmosphere, is the second most abundant constituent responsible for climatic forcing after carbon dioxide [1, 2]. $\mathrm{CH}_{4}$ emissions above $90 \%$ originate from leakage of natural gas (NG), oil and gas storage, transportation and distribution systems. These activities increase atmospheric $\mathrm{CH}_{4}$ concentration levels and lead to serious climate changes, which must be addressed [3-7]. The earth's atmospheric $\mathrm{CH}_{4}$ concentration has increased by $\sim 150 \%$ since 1750 , and accounts for $20 \%$ of the total radiative forcing from greenhouse gases. $\mathrm{CH}_{4}$ is also an industrial safety hazard, especially in the coal mining industry and in the handling of liquefied $\mathrm{CH}_{4}$. Hence the development of a real-time, portable, reliable sensor system for monitoring $\mathrm{CH}_{4}$ concentration level in urban and rural areas is important [8-12].

In comparison with mass spectrometry or gas chromatography, optical methods based on infrared laser spectroscopy [13-17] are advantageous for $\mathrm{CH}_{4}$ sensing in terms of size, time resolution and cost and require no pretreatment and/or accumulation of the concentration of the targeted gas samples. Tunable infrared laser absorption spectroscopy (TLAS) [18-20] enables non-contact measurements and has proven to be an excellent tool for trace gas detection in various applications. TLAS requires a tunable laser capable of near or mid-infrared single frequency emission with a narrow linewidth at the targeted absorption line of a gas molecule in order to achieve high detection sensitivity and selectivity. Gallium antimonite (GaSb)-based interband cascade lasers (ICLs) provide continuous wave (CW) radiation between $3.0 \mu \mathrm{m}$ and $6.0 \mu \mathrm{m}$ at room temperature, which initiated a new pathway for mid-infrared sensing [21-23]. Both single-mode and multi-mode ICLs have been adopted in trace gas detection in recent years [24-26]. Since the emission wavelength range of 3-6 $\mu \mathrm{m}$ of an interband cascade laser (ICL) covers the strong fundamental absorption band of $\mathrm{CH}_{4}$ at $3.3 \mu \mathrm{m}$, a low limit of detection (LoD) in parts per billion by volume (ppbv) level can be achieved for an ICL-based sensor in the 3-6 $\mu \mathrm{m}$ spectral range using TLAS. Such a sensor system is more advantageous in atmospheric $\mathrm{CH}_{4}$ detection than light emitting diode (LED) based $\mathrm{CH}_{4}$ sensors with a LoD of tens of ppmv [27, 28].

In previous studies, an ICL based $\mathrm{CH}_{4}$ sensor based on an absorption line at $3038.5 \mathrm{~cm}^{-1}$ and a laser 
direct absorption spectroscopy (LDAS) technique [29] as well as a single-ICL based dual-gas $\mathrm{CH}_{4} / \mathrm{C}_{2} \mathrm{H}_{6}$ sensor system based on a $\mathrm{CH}_{4}$ absorption line at $2999.06 \mathrm{~cm}^{-1}$ and wavelength modulation technique (WMS) were developed and demonstrated [30]. Several field campaigns were conducted using these sensors for the monitoring of $\mathrm{CH}_{4}$ leakage at natural gas vehicle fueling stations in the Greater Houston Area. However, the power requirements of these sensor systems was relatively high $(\sim 250 \mathrm{~W})$, mainly due to the use of an oil-free vacuum pump (KNF Neuberger Inc., model N 813.5 ANE/AF, with $100 \mathrm{~W}$ in power consumption and $7.5 \mathrm{~kg}$ in weight), a pressure controller \& readout (MKS Instruments, Inc., Type $649 \& 167 \mathrm{~A}, \sim 8 \mathrm{~W}$ in power consumption and $2.0 \mathrm{~kg}$ in weight), an ICL current driver (Thorlabs, model LDC $202 \mathrm{C}$, with a $\sim 25 \mathrm{~W}$ power consumption and $\sim 3.1 \mathrm{~kg}$ in weight) as well as a temperature controller (Thorlabs, model TED 200C, $\sim 60 \mathrm{~W}$ in power consumption and $\sim 3.1 \mathrm{~kg}$ in weight). This equipment required a large-sized vehicle for field deployment of such a $\mathrm{CH}_{4}$ sensor system. Hence a more portable $\mathrm{CH}_{4}$ sensor system was developed to address these power requirements and size limitations. A DC pump (KNF Neuberger Inc., model UN85.3 KNDC) and custom board-level electronics (a laser driver $(5 \times 4.3$ $\mathrm{cm})$ and a temperature controller $(4.5 \times 4 \mathrm{~cm}))$ were utilized, leading to a significant reduction in both size and power-consumption of the sensor system. Furthermore, the pressure inside the gas cell was measured by means of direct Lorentzian absorption fitting instead of a commercial pressure controller. Precise compensation of $\mathrm{CH}_{4}$ concentration changes resulting from pressure variations was performed. The performance of the $\mathrm{CH}_{4}$ sensor system was confirmed to be applicable for pressure conditions ranging from 25 to 800 Torr for $\mathrm{CH}_{4}$ detection based on laboratory and field deployment measurements.

\section{2. $\mathrm{CH}_{4}$ sensor configuration and design details}

\subsection{Optimum $\mathrm{CH}_{4}$ line selection}

$\mathrm{CH}_{4}$ has a strong fundamental absorption band in the mid-infrared spectral range centered at $~ 3.3$ $\mu$ m. HITRAN absorption spectra of $2 \mathrm{ppmv} \mathrm{CH}_{4}$ and $2 \% \mathrm{H}_{2} \mathrm{O}$ (relative humidity 60\% @ $298 \mathrm{~K}$ ) calculated at 700, 300 and 100-Torr gas pressures using a 5460-cm effective optical path length are depicted in Fig. A1(a) (Here 'A' means this figure is presented in the Appendix. The same meanings for

Figs. A2-A6 below). A strong $\mathrm{CH}_{4}$ absorption line was found to be located at $3038.5 \mathrm{~cm}^{-1}$ with a line 
intensity of $8.958 \times 10^{-20} \mathrm{~cm} /$ molecule. A nearby $\mathrm{H}_{2} \mathrm{O}$ absorption line at $3037.6 \mathrm{~cm}^{-1}$ is relatively flat near $3038.5 \mathrm{~cm}^{-1}$ at concentration levels of $<2 \%$, so that the $\mathrm{H}_{2} \mathrm{O}$ absorption can be treated as background information in data processing. In addition, a calcium sulfate $\mathrm{H}_{2} \mathrm{O}$ trap (W.A. Hammond Drierite, CAS \#7778-18-9, i.e. the drier shown in Fig. 1) was used in this sensor system to further reduce $\mathrm{H}_{2} \mathrm{O}$ concentration to as low as $0.1 \%$ and minimize the effect of $\mathrm{H}_{2} \mathrm{O}$ on $\mathrm{CH}_{4}$ detection. The $\mathrm{CH}_{4}$ linewidth is reduced as the pressure decreases and can be utilized for pressure measurements.

A commercially available ICL from Nanoplus, mounted in a TO66 header was attached to a heat sink with a thermoelectric cooler (TEC, physical size: $5 \times 5 \times 5 \mathrm{~cm}$ ). The ICL output power was measured to be $\sim 1.9 \mathrm{~mW}$, when the ICL operates at a temperature of $\sim 30{ }^{\circ} \mathrm{C}$ and a driving current of $54 \mathrm{~mA}$. The ICL wavenumber can be tuned between $3034 \mathrm{~cm}^{-1}$ and $3042 \mathrm{~cm}^{-1}$. The experimentally determined current and temperature tuning coefficient of this ICL are $-0.232 \mathrm{~cm}^{-1} / \mathrm{mA}$ and $-0.240 \mathrm{~cm}^{-1} /{ }^{\circ} \mathrm{C}$, respectively. An ICL injection current of $40 \mathrm{~mA}$ and a $30.95{ }^{\circ} \mathrm{C}$ operating temperature were selected for $\mathrm{CH}_{4}$ concentration measurements at the optimum targeted absorption line of $3038.5 \mathrm{~cm}^{-1}$, as shown in Fig. A1(b).

\subsection{Sensor configuration}

The mid-infrared $\mathrm{CH}_{4}$ sensor architecture is shown in Fig. 1, which consists of an optical and an electrical sub-system. A $3291 \mathrm{~nm}$ CW, DFB ICL was used as the infrared source in the optical sub-system. The laser beam was coupled into a mode matching lens (L), reflected by two plane mirrors (M3 and M4), and entered the MPGC with a $54.60 \mathrm{~m}$ optical path length. After $\sim 435$ reflections, the output beam was focused onto a TEC mercury-cadmium-telluride (MCT) photodetector (VIGO System, model PVI-4TE-4) using a parabolic mirror (PM).

The electrical part of the sensor system consists of a laptop (Dell, model \# PP04X), a DAQ card (National Instrument, model USB-6356), a custom board-level laser current driver and a temperature controller. The laser driver and temperature controller both have a compact size of $<5 \times 5 \mathrm{~cm}$ and a supply voltage of $+12 \mathrm{~V}$. The temperature controller is capable of operating with an accuracy of $< \pm 0.001{ }^{\circ} \mathrm{C}$ with a stable temperature drive current. The ratio between input voltage and output current was adjusted to $\sim 2.14 \mathrm{~mA} / \mathrm{V}$ to improve the current accuracy, which was 10 times more sensitive than commercial 
drivers with an I/V relation of $\sim 20 \mathrm{~mA} / \mathrm{V}$. The power consumption of the two drivers is $<1.5 \mathrm{~W}$, which is also significantly lower than commercial products (up to $\sim 80 \mathrm{~W}$ in total). A LDAS technique was used for $\mathrm{CH}_{4}$ detection, which only requires a saw tooth scan signal to drive the ICL. This scan signal was generated by a LabVIEW-controlled DAQ card. The MCT detector signal was sent to the DAQ card for data acquisition, triggered by a signal generation module. A LabVIEW based signal-processing system was developed and used to fit the absorption peak (LAF), measure the pressure (PD), perform pressure compensation (PC), and determine the concentration (CD). A compact DC pump (KNF Neuberger Inc., model UN 85.3 KNDC) was used to pump the target gas into the MPGC.

A standard gas dilution system (Environics, Series 4040) was used for calibration and performance assessment of the $\mathrm{CH}_{4}$ sensor. A 2.1 ppmv (balanced by nitrogen $\left(\mathrm{N}_{2}\right)$ ) $\mathrm{CH}_{4}$ cylinder and a pure $\mathrm{N}_{2}$ cylinder were used as input cylinders to the dilution system for the preparation of $\mathrm{CH}_{4}$ samples with different concentration levels (ranging from ppbv to ppmv). Our data-processing routine was based on LabVIEW.

\subsection{LabVIEW-based data processing system}

The function diagram of the LabVIEW based laptop platform is shown in Fig. A2. There are three main functions of this platform: signal generation (SG), signal acquisition (SA) and absorption fitting \& processing. For the SG sub-system, a scan signal array was generated and supplied to a digital-to-analog converter (DAC) module. The drive signal was applied to the ICL via the DAQ card. For the SA, via the use of an analog-to-digital converter (ADC), the output signal from the MCT detector was sampled at the same sampling rate with a DAC. $N$ frames of spectra were sampled per one loop. The sampled $N$ frames were then averaged in order to suppress random noise based on absorption fitting and processing. The background signal was obtained via a fifth-order polynomial fitting using the spectral data without $\mathrm{CH}_{4}$ absorption (i.e. excluding the absorption region). Following normalization on the absorption signal, a Lorentzian absorption fitting was performed, and both the absorbance and the full width at the half maximum (FWHM) of the absorption line were derived. The pressure was determined based on the FWHM and used to compensate the absorbance changes resulting from pressure variations. Finally, the 
concentration was calculated using the compensated absorbance as described in Section 3.

\section{Pressure measurement based on spectral line broadening}

\subsection{Lorentzian absorption fitting for pressure measurements}

A spectroscopic transition of the $\mathrm{CH}_{4}$ molecule is associated with a specific amount of energy. When this energy is measured by means of a spectroscopic technique, the spectroscopic line has a particular lineshape. Numerous factors can contribute to the broadening of spectral lines. The principal sources of broadening are: lifetime broadening, Doppler broadening, pressure broadening and collisional broadening. Spectral lineshapes and line widths can also be affected by instrumental factors, which can be described by a convolution of the intrinsic line shape with instrument transfer function. These mechanisms can act separately or in combination. If each effect is independent of the other, the observed line profile is a convolution of the line profiles of each mechanism. For example, a combination of Doppler and pressure broadening effects results in a Voigt profile. For molecules in the gas phase, the principal effects are Doppler and pressure broadening, which apply to rotational spectroscopy, rotational-vibrational spectroscopy and vibronic spectroscopy. In this paper, the pressure broadening effect, which yields a Lorentzian profile, is discussed.

In terms of signal processing, $N$ frames of the output signal from the detector $u_{\mathrm{r}}(t)$ were sampled during each calculation loop by means of the DAQ card and averaged as

$$
u_{\mathrm{r}, \text { avr }}(t)=\frac{1}{N} \sum_{i=1 \sim N} u_{\mathrm{r}, i}(t)=\underbrace{u_{\mathrm{r}, \text { avr }}(t)}_{\text {background }}-\underbrace{u_{\mathrm{r}, \text { avr }}(t)}_{\text {absorption }}
$$

Once $u_{\mathrm{r}, \text { avr }}(t)$ was obtained, data fitting based on LabVIEW was used to obtain the background signal

$$
u_{\mathrm{r}, \mathrm{bac}}(t)=\underbrace{u_{\mathrm{r}, \text { avr }}(t)}_{\text {background }}
$$

Furthermore, the following processing was performed to eliminate the background signal

$$
u_{\mathrm{r}, \mathrm{final}}(t)=\frac{u_{\mathrm{r}, \mathrm{bac}}(t)-u_{\mathrm{r}, \text { avr }}(t)}{u_{\mathrm{r}, \mathrm{bac}}(t)}
$$

Then the absorbance value is derived 


$$
u_{\mathrm{r}, \text { absorbance }}(t)=-\ln \left[1-u_{\mathrm{r}, \text { final }}(t)\right]
$$

With pressure broadening, $u_{\mathrm{r}, \text { final }}(t)$ can be fitted by a Lorentzian signal as

$$
u_{\mathrm{r}, \text { lorentzian }}(t)=\frac{A}{1+4\left(\frac{t-t_{0}}{\mathrm{FWHM}}\right)} \stackrel{\text { lorentzian absorption fitting }}{\longrightarrow} u_{\mathrm{r}, \text { absorbance }}(t)
$$

where $A$ represents the absorption intensity, and $t_{0}$ is the central absorption peak position.

As an example, the absorption spectra obtained from a 2.1-ppm $\mathrm{CH}_{4}$ are shown in Fig. A3(a). In this experiment, the pressure inside the gas cell was set to 700 Torr by means of a pressure controller (MKS, Type 640). A ramp scan signal with an amplitude of $\sim 3.27 \mathrm{~V}$ and a frequency of $500 \mathrm{~Hz}$ was supplied to the custom laser driver for generating ICL drive currents from 38 to $45 \mathrm{~mA}$. The sampling rate of the sensing signal was $1 \mathrm{MHz}$ leading to 2000 data points per scan period. The red dotted line is a 50 -frame averaged absorption signal and the red solid line is the Lorentzian fitting signal. During data processing, the first 200 data points were removed because of fluctuations caused by the falling edge of the ramp signal. Similarly, we obtained the Lorentzian fitted absorption lines corresponding to different pressures from 100 Torr to 800 Torr, as shown in Fig. A3(b).

\subsection{Pressure calibration using a 2.1-ppmv $\mathrm{CH}_{4}$ sample}

During pressure calibration, the laser temperature was set to $30.95^{\circ} \mathrm{C}$ using a custom TEC driver. For high-pressure measurements, the laser driver current was set to cover from 38 to $45 \mathrm{~mA}$ (corresponding to a wavenumber range of $1.624 \mathrm{~cm}^{-1}$ ) to scan the $\mathrm{CH}_{4}$ absorption line at $3038.5 \mathrm{~cm}^{-1}$. This required a ramp scan signal with an amplitude of $\sim 3.27 \mathrm{~V}$ to be applied to the custom laser driver. For low-pressure measurements, the laser driver current was set to $39.8-42.2 \mathrm{~mA}$ (corresponding to a wavenumber range of $0.5616 \mathrm{~cm}^{-1}$ ), which required a ramp scan signal with an amplitude of $\sim 1.12 \mathrm{~V}$. Data sampling was triggered by the ramp signal to realize a complete sample period of the sensing signal, which contains 2000 points. $N=50$ frames were sampled per one calculation loop. All the data were recorded by a Dell computer (model \# PP04X) for processing and post-analysis.

For the high pressure range of 100-800 Torr, the calibration was carried out based on pressure 
measurements using a standard 2.1-ppmv $\mathrm{CH}_{4}$ sample. The FWHM value was recorded for $\sim 10$ min for each gas pressure (at 100-Torr intervals), as shown in Fig. 2(a). The FWHM value for each pressure was then averaged and plotted as a function of the pressure as depicted in Fig. 2(b). The theoretical FWHM values obtained from a HITRAN 2012 simulation were also added in Fig. 2(b) as a comparison with experimental results. The two group values agree well within this range. The relationship between the pressure and the FWHM within a pressure range of 100-800 Torr can be represented by a linear curve as

$$
P=6162.97 \mathrm{FWHM}-38.54, P \in[100,800 \text { Torr })
$$

Another calibration was carried out using the same standard 2.1-ppmv $\mathrm{CH}_{4}$ sample for a low pressure range of from 25 to100 Torr, as shown in Fig. 2(c). Fig. 2(d) shows the relation between the averaged FWHM and pressure. This relationship was fitted by a linear curve as

$$
P=8026.23 \mathrm{FWHM}-68.61, \quad P \in[0,100 \text { Torr })
$$

For this pressure range, the measured FWHM values were also in agreement with theoretical results.

\subsection{Dynamic pressure measurements and Allan deviation}

Three other $\mathrm{CH}_{4}$ samples with concentration levels of 1.3, 1.5 and 1.7 ppmv generated by the gas dilution system, were used to measure the pressure inside the gas cell. The pressure was controlled by using the MKS pressure controller and switched for dynamic testing. The time series of the measured pressures $\left(60,100,300\right.$ and 500 Torr) are shown in Fig. A4(a), where each $\mathrm{CH}_{4}$ sample was tested for $30 \mathrm{~min}$ for the four pressures. The measured results agree well with the theoretical values, indicating a satisfactory performance of pressure measurements.

The noise level of pressure measurements was determined by passing the 2.1-ppm $\mathrm{CH}_{4}$ sample into the gas cell with a controlled pressure of 700 Torr and subsequent monitoring of the FWHM. The FWHM was transformed to pressure based on the fitting relation of Eq. (6). A pressure measurement was performed over a time period of $\sim 40 \mathrm{~min}$, as shown in Fig. A4(b). An average pressure of $\sim 695.9 \pm 3.8$ Torr $(1 \sigma)$ was measured for the 40-min observation time. The Allan deviation was plotted on a log-log scale versus averaging time, $\tau$, as shown in Fig. A4(c). The plot indicates a measurement precision of $\sim 1.65$ 
Torr with $\mathrm{a} \sim 2.2 \mathrm{~s}$ averaging time. However, the Allan-Werle plot is flat with increasing the averaging time, though it shows a minimum value of $\sim 0.99$ Torr with an averaging time of $44 \mathrm{~s}$. The flat curve indicates that the pressure measurement system was not dominated by white noise in comparison with the ideal decreasing line $(\sim 1 /$ sqrt $(\tau))$ of a white-noise dominated system. Uncertainties resulting from both measurement system (including DAQ card, ICL, detector, data-processing software) and pressure calibration system (MKS pressure controller) are responsible for the pressure measurement precision.

\section{4. $\mathrm{CH}_{4}$ sensor performance with pressure measurement/compensation}

\subsection{Pressure-compensation method}

Experiments were performed to measure the relation between the absorbance and the pressure for a standard 2.1-ppm $\mathrm{CH}_{4}$ sample, which was used as the "calibration gas". The pressure inside the gas cell was controlled at specific levels using the MKS pressure controller. For a low (30-90 Torr) and a high (100-800 Torr) pressure range, the measured absorbances are shown in Fig. 3. Incremental fitting was used to obtain their relation, which can be represented by a second order polynomial curve within the low pressure range of $0-100$ Torr and a fifth-order polynomial curve in the high pressure range of $100-800$ Torr. The obtained fitting curves are:

$$
\alpha(P, 2.1 \mathrm{ppm})=\left\{\begin{array}{l}
0.01428+0.00144 P-6.96693 P^{2}, P \in[0,100 \text { Torr }) \\
0.05545+5.58098 \times 10^{-4} P-2.63225 \times 10^{-6} P^{2}+ \\
6.03593 \times 10^{-9} P^{3}-6.68592 \times 10^{-12} P^{4}+2.84424 \times 10^{-15} P^{5}, P \in[100,800 \text { Torr })
\end{array}\right.
$$

For an unknown concentration $C$ inside the gas cell, both pressure $P$ and absorbance $\alpha(P, C)$ can be achieved by means of Lorentzian absorption fitting of the absorption peak. Since $\alpha(P, C) \propto C$, we have the following relation at a pressure $P$ as

$$
\frac{\alpha(P, C)}{\alpha(P, 2.1 \mathrm{ppm})}=\frac{C}{2.1 \mathrm{ppm}}
$$

Therefore, we can derive the unknown $\mathrm{CH}_{4}$ concentration with the compensation as 


$$
C_{\text {com }}=\frac{\alpha(P, C)}{\alpha(P, 2.1 \mathrm{ppm})} \times 2.1 \mathrm{ppm}
$$

Without compensation, the measured concentration can be determined as

$$
C_{\text {uncom }}=\frac{\alpha(P, C)}{\left.\alpha_{0}\right|_{P_{0}=700 \text { Torr }, C_{0}=2.1 \mathrm{ppm}}} \times 2.1 \mathrm{ppm}
$$

where $\alpha_{0}$ is a definite absorbance of the 2.1-ppm $\mathrm{CH}_{4}$ at 700-Torr pressure .

\subsection{Sensor performance using a standard 2.1-ppmv $\mathrm{CH}_{4}$ sample}

A 2.1-ppmv $\mathrm{CH}_{4}$ sample was passed through the MPGC. The gas pressure was decreased from 700 Torr to 30 Torr by using the MKS pressure controller. The pressure inside the MPGC, the $\mathrm{CH}_{4}$ concentration with compensation using Eq. (10), and the $\mathrm{CH}_{4}$ concentration without compensation using Eq. (11) were measured. The time series of the results are presented in Fig. A5. Without pressure compensation, the measured $\mathrm{CH}_{4}$ concentration level decreased from $2.10 \mathrm{ppm}$ to $1.06 \mathrm{ppm}$ as the pressure decreases from 700 Torr to 30 Torr, which illustrates that a pressure change has an impact on the concentration. However, after a pressure compensation based on Eq. (10), the measured concentration becomes stable at $~ 2.1 \mathrm{ppm}$ in a pressure range of from 700 to 60 Torr. However, large fluctuations occur at low pressures of 40 and 30 Torr, due to the gas flow in the gas cell causing strong variations in optical beam propagation and an unstable mode pattern inside the gas cell.

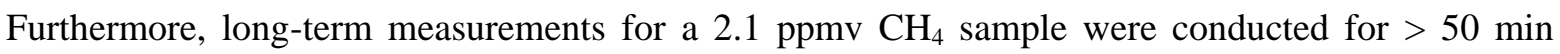
under a controlled pressure of 700 Torr with a sampling period of $2.5 \mathrm{~s}$. The measured pressure and $\mathrm{CH}_{4}$ concentrations with/without compensation are shown in Fig. A6(a). The average $\mathrm{CH}_{4}$ concentration levels for the above two cases are $\sim 2.10 \mathrm{ppm} \pm 11.0 \mathrm{ppb}(1 \sigma)$ and $\sim 2.10 \mathrm{ppm} \pm 10.7 \mathrm{ppb}(1 \sigma)$ for a 50 -min observation time. Allan deviation plots were obtained and are shown in Fig. A6(b). The Allan deviations are $2.66 \mathrm{ppb}$ without compensation and $2.25 \mathrm{ppb}$ with compensation for an averaging time of $2.5 \mathrm{~s}$. There is a decrease of $41 \mathrm{ppt}$ in sensor stability as a result of pressure compensation. The sensor has its optimum stability for an averaging time of $\sim 13 \mathrm{~s}$ according to the plot in Fig. A6(b).

\subsection{Sensor performance using diluted $\mathrm{CH}_{4}$ samples}


The sensor performance was further investigated using five diluted $\mathrm{CH}_{4}$ samples with concentration levels of 1.0, 1.2, 1.4, 1.6 and $2.1 \mathrm{ppm}$. Four groups of measurements were conducted at pressures of 60, 100, 300 and 500 Torr. For each pressure, the $\mathrm{CH}_{4}$ concentration was increased from 1.0 ppm to $2.1 \mathrm{ppm}$. The measured $\mathrm{CH}_{4}$ concentration levels with/without compensation were recorded during the whole monitoring period of 200 minutes as shown in Fig. 4. The compensated $\mathrm{CH}_{4}$ concentration agrees well with the standard value by eliminating the error caused by pressure variations. At low pressure of 60 Torr, there are both pressure and concentration fluctuations and despite this, the measured concentration levels are in good agreement with the actual concentration levels of the diluted $\mathrm{CH}_{4}$ samples.

\section{Atmospheric $\mathrm{CH}_{4}$ detection}

\subsection{Indoor measurements}

The performance of the pressure-independent sensor system was evaluated based onCH $\mathrm{H}_{4}$ concentration measurements in a laboratory environment in the Rice Laser Science Laboratory, Suite SST 104 on the Rice University campus. Fig. 5 shows the measured $\mathrm{CH}_{4}$ concentration levels from 17:30 CDT, Aug 06, 2016 to 09:10 CDT, Aug 08, 2016. The average $\mathrm{CH}_{4}$ concentration levels with/without compensation were $\sim 2.010 \pm 0.171 \mathrm{ppmv}(1 \sigma)$ and $\sim 2.009 \pm 0.173$ ppmv, respectively, and the average pressure was $\sim 730 \pm 12$ Torr $(1 \sigma)$. The $\mathrm{CH}_{4}$ concentration exhibited relatively minor variations during the period of monitoring, with a slight increase during the early morning hours followed by decreasing concentrations during the day, as observed by previous studies [29]. Some peaks occurred during Sunday night, probably due to indoor and outdoor air exchange.

\subsection{Outdoor measurements on Rice University campus}

The sensor system was also evaluated for the detection of atmospheric $\mathrm{CH}_{4}$ on the Rice University campus. For outdoor measurements, the dual trace gas sensor system was mounted on a cart and power was supplied by a battery (Power-Sonic, model PG-12V150-FR) connected to an AC inverter (Wagan Tech, model 9622). The photograph, shown in Fig. 6(a), of the sensor system was taken when the cart was ready to be placed outside the Laser Science Laboratory (SST 104) to monitor variations of atmospheric $\mathrm{CH}_{4}$ concentration levels. For continuous day and night monitoring, the cart was placed inside the 
laboratory and the outside air was pumped into the gas cell using a long sampling line. The measured concentrations are plotted in Fig. 6(b). The experiment was conducted from 11:00 CDT on June 30, 2016 to 11:00 CDT on July 6, 2016 ( 144 hours sampling). Fluctuations in concentration levels were observed during atmospheric monitoring of $\mathrm{CH}_{4}$. The $\mathrm{CH}_{4}$ concentration without compensation varied from 1.87 to 5.57 ppmv, with an average value of $2.29 \pm 0.40 \mathrm{ppmv}(1 \sigma)$. The $\mathrm{CH}_{4}$ concentration with compensation varied from 1.89 to $5.40 \mathrm{ppmv}$, with an average value of $2.29 \pm 0.38 \mathrm{ppmv}(1 \sigma)$. The measured pressure shows an average value of $760 \pm 17$ Torr. The average pressure inside the gas cell is nearly equal to the ambient pressure due to the small air-flow. There were obvious peaks in $\mathrm{CH}_{4}$ concentrations during the early morning hours and then dropped gradually to its typical urban background level of $\sim 1.9-2.2$ ppmv. The detected $\mathrm{CH}_{4}$ concentration levels exhibited a typical hourly profile expected for this gas species which is related to boundary layer dynamics and the extent of mixing in the atmosphere [31].

\subsection{Field campaign in Houston, $T X$}

The $\mathrm{CH}_{4}$ sensor system was deployed in a vehicle to evaluate its performance for atmospheric $\mathrm{CH}_{4}$ monitoring in a comprehensive field campaign. The sensor system was driven from the Rice University campus monitoring $\mathrm{CH}_{4}$ for a round-trip distance of $\sim 12$ miles between 12:00 pm - 12:38 pm CDT on August 13, 2016. During this campaign, a globe positioning system (GPS, Adafruit, Version 3) continuously recorded the position of the sensor system. The data (i.e. $\mathrm{CH}_{4}$ mixing ratio and GPS coordinates) during the field test were recorded every 2.5 s. Fig. 7(a) shows a photograph of the vehicle with the $\mathrm{CH}_{4}$ sensor system placed in its trunk. Two maps were drawn to show the measured $\mathrm{CH}_{4}$ concentration and pressure during each trip based on the measured data,. The maps in Figs. 7(b) and 7(c) show the pressure and $\mathrm{CH}_{4}$ concentration levels detected along the driving path during the field trip. The average pressure inside the MPGC is below the ambient pressure because of a relatively large gas flow, which shows an average value of $746.7 \pm 6.54$ Torr $(1 \sigma)$. The measured $\mathrm{CH}_{4}$ concentration with compensation varied from 1.95 to $2.02 \mathrm{ppmv}$, with an average value of $1.99 \mathrm{ppmv} \pm 11.4 \mathrm{ppbv}(1 \sigma)$.

\section{Conclusions}

A mid-infrared $\mathrm{CH}_{4}$ sensor system without pressure control was developed using a $3.291 \mu \mathrm{m}$ TEC, 
CW ICL and a dense patterned MPGC with an effective $54.6 \mathrm{~m}$ optical path length. The ICL targeted a strong $\mathrm{CH}_{4}$ absorption line at $3038.5 \mathrm{~cm}^{-1}$ in the fundamental absorption band of $\mathrm{CH}_{4}$. The pressure inside the MPGC was measured based on direct Lorentzian absorption line fitting. Pressure calibration was performed from 25 to 800 Torr using a 2.1-ppmv $\mathrm{CH}_{4}$ sample. Pressure measurements of 1.3, 1.5 and 1.7-ppmv $\mathrm{CH}_{4}$ samples at different pressures of $60,100,300$ and 500 Torr were performed after calibration. An Allan deviation analysis of the measured pressure of a 2.1-ppmv $\mathrm{CH}_{4}$ at 700-Torr pressure indicates a precision of $\sim 1.65$ Torr with a 2.5-s averaging time. Compensation of $\mathrm{CH}_{4}$ concentration changes resulting from pressure variations was used to obtain accurate $\mathrm{CH}_{4}$ concentration levels. Concentration measurements of a 2.1-ppmv $\mathrm{CH}_{4}$ sample at a 700-Torr pressure yielded an Allan deviation of $2.25 \mathrm{ppbv}$ for an averaging time of $2.5 \mathrm{~s}$. By varying the pressure from 25 to 800 Torr, the reported $\mathrm{CH}_{4}$ sensor operates normally on $\mathrm{CH}_{4}$ samples with concentration levels of 1.0, 1.2, 1.4, 1.6 and 2.1 ppmv. Measurements for both indoor and outdoor concentration $\mathrm{CH}_{4}$ changes were carried out on the Rice University campus. A field test in Houston, TX was also conducted to evaluate the performance of the sensor system as a robust and reliable field-deployable sensor system for atmospheric monitoring. The reported ICL-based sensor platform has wide applications for atmospheric $\mathrm{CH}_{4}$ measurements with advantages of reduced size, weight and cost as compared to a pressure-controlled gas sensor system.

\section{Acknowledgments}

The authors wish to acknowledge the support from National Science Foundation (NSF) (ERC MIRTHE award), USA Robert Welch Foundation (C-0586), NSF Phase II SBIR (IIP-1230427DE DE), DOE ARPA-E awards (DE-0000545, DE-0000547), National Natural Science Foundation of China (NSFC) (61627823, 61307124, 61575113, 61275213), Changchun Municipal Science and Technology Bureau (14KG022), High School Outstanding Young Teacher Training Program of Guangdong Province (YQ2015071), and China Scholarship Council (201506175025, 201508440112). 


\section{References}

[1] B. Heiko, G.F. France, G. T. Charles, R. S. Clare, J. E. Tim, M. Ian, S. Anatoly, N. Sten, S. Anatoly, O. Alexander, and S. Christiane, Impact of the Arctic Oscillation pattern on interannual forest fire variability in Central Siberia, Geophys. Res. Lett. 32 (2005) L14709.

[2] I.J. Simpson, F.S. Rowland, S. Meinardi, and D.R. Blake, Influence of biomass burning during recent fluctuations in the slow growth of global tropospheric methane, Geophys. Res. Lett. 33 (2006) L22808.

[3] G. Pétron, G. Frost, B. R. Miller, A. I. Hirsch, S. A. Montzka, A. Karion, M. Trainer, C. Sweeney, A. E. Andrews, L. Miller, J. Kofler, A. Bar-Ilan, E. J. Dlugokencky, L. Patrick, C. T. Moore Jr., T. B. Ryerson, C. Siso, W. Kolodzey, P. M. Lang, T. Conway, P. Novelli, K. Masarie, B. Hall, D. Guenther, D. Kitzis, J. Miller, D. Welsh, D. Wolfe, W. Neff, and P. Tans, Hydrocarbon emissions characterization in the Colorado Front Range: A pilot study, J. Geophys. Res. 117 (2012) D04304.

[4] A. Karion, C. Sweeney, G. Pétron, G. Frost, R. M. Hardesty, J. Kofler, B. R. Miller, T. Newberger, S. Wolter, R. Banta, A. Brewer, E. Dlugokencky, P. Lang, S. A. Montzka, R. Schnell, P. Tans, M. Trainer, R. Zamora, and S. Conley, Methane emissions estimate from airborne measurements over a western United States natural gas field, Geophys. Res. Lett. 40 (2013) 4393-4397.

[5] S. M. Millera, S. C. Wofsya, A. M. Michalakb, E. A. Kortc, A. E. Andrewsd, S. C. Biraude, E. J. Dlugokenckyd, J. Eluszkiewiczf, M. L. Fischerg, G. J. Maenhouth, B. R. Milleri, J. B. Milleri, S. A. Montzkad, T. Nehrkornf, and C. Sweeneyi, Anthropogenic emissions of methane in the United States, Proc. Natl. Acad. Sci. U.S A. 110 (2013) 20018-20022.

[6] A. R. Brandt, G. A. Heath, E. A. Kort, F. O'Sullivan, G. Pétron, S. M. Jordaan, P. Tans, J. Wilcox, A. M. Gopstein, D. Arent, S. Wofsy, N. J. Brown, R. Bradley, G. D. Stucky, D. Eardley, and R. Harriss, Methane Leaks from North American Natural Gas Systems, Science 343 (2014) 733-735.

[7] S. Schwietzke, W. M. Griffin, S. Matthews, and L. M. P. Bruhwiler, Natural Gas Fugitive Emissions Rates Constrained by Global Atmospheric Methane and Ethane, Environ. Sci. Technol. 48 (2014) $7714-7722$. 
[8] L. Dong, J. Wright, B. Peters, B.A. Ferguson, F.K. Tittel, and S. McWhorter, Compact QEPAS sensor for trace methane and ammonia detection in impure hydrogen, Appl. Phys. B-Lasers Opt. 107 (2012) $459-467$.

[9] J. Leis, D. Buttsworth, C. Snook, G. Holmes, Detection of potentially explosive methane levels using a solid-state infrared source, IEEE Trans. Instrum. Meas. 63 (2014) 3088-3095.

[10] M. Triki, T. Nguyen Ba, and A. Vicet, Compact sensor for methane detection in the mid infrared region based on quartz enhanced photoacoustic spectroscopy, Infrared Phys. Technol. 69 (2015) $74-80$.

[11] M. Köhring, S. Huang, M. Jahjah, W. Jiang, W. Ren, U. Willer, C. Caneba, L. Yang, D. Nagrath, W. Schade, and F. K. Tittel, QCL-based TDLAS sensor for detection of NO toward emission measurements from ovarian cancer cells, Appl. Phys. B-Lasers Opt.117 (2014) 445.

[12] W. Ren, L. Luo, and F. K. Tittel, Sensitive detection of formaldehyde using an interband cascade laser near 3.6 $\mu \mathrm{m}$, Sens.Actuators, B: Chem. 221 (2015) 1062.

[13] D.G. Lancaster, R. Weidner, D. Richter, F.K. Tittel, and J. Limpert, Compact $\mathrm{CH}_{4}$ sensor based on difference frequency mixing of diode lasers in quasi-phasematched LiNbO3, Opt. Commun. 175 (2000) 461-468.

[14] D.G. Lancaster, and J. M. Dawes, Methane detection with a narrow-band source at $3.4 \mu \mathrm{m}$ based on a Nd: YAG pump laser and a combination of stimulated Raman scattering and difference frequency mixing, Appl. Opt. 35 (1996) 4041-4045.

[15] C. Fischer, and M.W. Sigrist, Trace-gas sensing in the $3.3-\mu \mathrm{m}$ region using a diode-based difference-frequency laser photoacoustic system, Appl. Phys. B-Lasers Opt. 75 (2002) 305-310.

[16] D. Richter, D.G. Lancaster, R.F. Curl, W. Neu, and F.K. Tittel, Compact mid-infrared trace gas sensor based on difference-frequency generation of two diode lasers in periodically poled LiNbO3, Appl. Phys. B-Lasers Opt. 67 (1998) 347-350.

[17] K.P. Petrov, S. Waltman, E.J. Dlugokencky, M. Arbore, M.M. Fejer, F.K. Tittel, and L.W. Hollberg, Precise measurement of methane in 3.4- $\mu \mathrm{m}$ difference-frequency generation in PPLN, Appl. Phys. 
B-Lasers Opt. 64 (1997) 567-572.

[18] J.A. Silver, Frequency-modulation spectroscopy for trace species detection: theory and comparison among experimental methods, Appl. Opt. 31 (1992) 707-717.

[19] P. Werle, A review of recent advances in semiconductor laser based gas monitors, Spectrochim. Acta. A 54 (1998) 197-236.

[20] S. Schilt, L. Thévenaz, and P. Robert, Wavelength modulation spectroscopy: combined frequency and intensity laser modulation, Appl. Opt. 42 (2003) 6728-6738.

[21] A. Joullie, and P. Christol, GaSb-based mid-infrared 2-5 $\mu$ m laser diodes, C. R. Phys. 4 (2003) $621-637$.

[22] M. Motyka, G. Sek, K. Ryczko, J. Misiewicz, T. Lehnhardt, S. Hoefling, and A. Forchel, Optical properties of GaSb-based type II quantum wells as the active region of midinfrared interband cascade lasers for gas sensing applications, Appl. Phys. Lett. 94 (2009) 251901.

[23] I. Vurgaftman, W. W. Bewley, C. L. Canedy, C. S. Kim, M. Kim, C. D. Merritt, J. Abell, J. R. Lindle, and J. R. Meyer, Rebalancing of internally generated carriers for mid-infrared interband cascade lasers with very low power consumption, Nature Commun. 2 (2011) 585.

[24] L. Dong, Y.J. Yu, C.G. Li, S. Stephen, and F.K. Tittel, Ppb-level formaldehyde detection using a CW room-temperature interband cascade laser and a miniature dense pattern multipass gas cell, Opt. Exp. 23 (2015) 19821-19830.

[25] K.M. Manfred, G.A.D. Ritchie, N. Lang, J. Ropcke, and J.H. van Helden, Optical feedback cavity-enhanced absorption spectroscopy with a $3.24 \mathrm{mu}$ m interband cascade laser, Appl. Phys. Lett. 106 (2015) 221106.

[26] J.H. Northern, S. O'Hagan, B. Fletcher, B. Gras, P. Ewart, C.S. Kim, M. Kim, C.D. Merritt, W.W. Bewley, C.L. Canedy, J. Abell, I. Vurgaftman, and J.R. Meyer, Mid-infrared multi-mode absorption spectroscopy using interband cascade lasers for multi-species sensing, Opt. Lett., 40 (2015) 4186-4189.

[27] W. L. Ye, C. T. Zheng, X. Yu, C. X. Zhao, Z. W. Song, and Y. D. Wang, Design and performances of 
a mid-infrared $\mathrm{CH} 4$ detection device with novel three-channel-based LS-FTF self-adaptive denoising structure, Sens. Actuators, B: Chem. 155 (2011) 37-45.

[28] C. T. Zheng, W. L. Ye, G. L. Li, X. Yu, C. X. Zhao, Z. W. Song, and Y. D. Wang, Performance enhancement of a mid-infrared $\mathrm{CH} 4$ detection sensor by optimizing an asymmetric ellipsoid gas-cell and reducing voltage-fluctuation: Theory, design and experiment, Sens. Actuators, B: Chem. 160 (2011) 389-398.

[29] L. Dong, C. Li, N. P. Sanchez, A. K. Gluszek, R. Griffin, and F. K. Tittel, Compact $\mathrm{CH}_{4}$ sensor system based on a continuous-wave, low power consumption, room temperature interband cascade laser, Appl. Phys. Lett. 108 (2016) 011106.

[30] W. Ye, C. Li, C. Zheng, N. P. Sanchez, A. K. Gluszek, A. J. Hudzikowski, L. Dong, R. J. Griffin, and F. K. Tittel, Mid-infrared dual-gas sensor for simultaneous detection of methane and ethane using a single continuous-wave interband cascade laser, Opt. Express 24(2016) 16973-16985.

[31] I. Bamberger, J. Stieger, N. Buchmann, and W. Eugster, Spatial variability of methane: Attributing atmospheric concentrations to emissions, Environ. Pollution, 190 (2014) 65-74. 


\section{Figure Captions}

Fig. 1. Schematic of a mid-infrared $\mathrm{CH}_{4}$ sensor without a pressure controller based on a single $\mathrm{CW}$, TEC ICL. ICL: interband cascade laser; DM: dichroic mirror; M: plane mirror; PM: parabolic mirror; MCT: mercury-cadmium-telluride; DAQ: data acquisition. In the LabVIEW platform on the laptop, SA: signal acquisition; LAF: Lorentzian absorption fitting; PD: pressure detection; PC: pressure compensation; CD: concentration decision; SG: signal generation.

Fig. 2. (a) Measured FWHM versus calibration time $t$ for eight pressures of $800,700,600,500,400,300$, 200, and 100 Torr. (b) Experimental data and fitting curve of gas pressure versus the averaged FWHM for pressures ranging from 100 to 800 Torr. (c) Measured FWHM versus calibration time $t$ for seven pressures of 100, 90, 80, 70, 60, 40, and 25 Torr. (d) Experimental data and fitting curve of pressure versus FWHM for a pressure range from 25 to 100 Torr. The red circle dots in Figs. 5(b) and 5(d) show the simulated FWHM for each pressure based on HITRAN 2012.

Fig. 3. Measured absorbance versus pressure for a 2.1-ppm $\mathrm{CH}_{4}$ sample within the pressure range of 0-800 Torr.

Fig. 4. Measured pressures and concentrations of five concentration levels of 1.0, 1.2, 1.4, 1.6 and 2.1 ppm atr four pressures of $60,100,300$ and 500 Torr. The data were recorded during a monitoring period $200 \mathrm{~min}$.

Fig. 5. Measured concentrations of $\mathrm{CH}_{4}$ in ambient air during $~ 40$ hours period on August 6-8, 2016 inside the Laser Science Laboratory (located in Space Science Technology building, Rice University).

Fig. 6. (a) A CW ICL based $\mathrm{CH}_{4}$ sensor system installed on a laboratory cart. (b) Measurement results of $\mathrm{CH}_{4}$ monitoring in the atmosphere for $\sim 144$ hours' time duration on the Rice University campus.

Fig. 7. (a) Photograph of the vehicle with the deployed $\mathrm{CH}_{4}$ sensor system parked at Rice University prior to a field test on August 13, 2016. (b) Measured pressure and (c) $\mathrm{CH}_{4}$ concentration levels along the route in the round-trip. The driving route consisted of: Rice University $\rightarrow$ Rice Blvd $\rightarrow$ Kirby $\mathrm{Dr} \rightarrow \mathrm{W}$ Holcombe Blvd $\rightarrow$ Stella Link Rd $\rightarrow$ S Brasewood Blvd $\rightarrow$ Linkwood Dr $\rightarrow$ Stella Link Rd $\rightarrow$ S Brasewood Blvd $\rightarrow$ Kirby Dr $\rightarrow$ Rice Blvd $\rightarrow$ Rice University. 
Figures

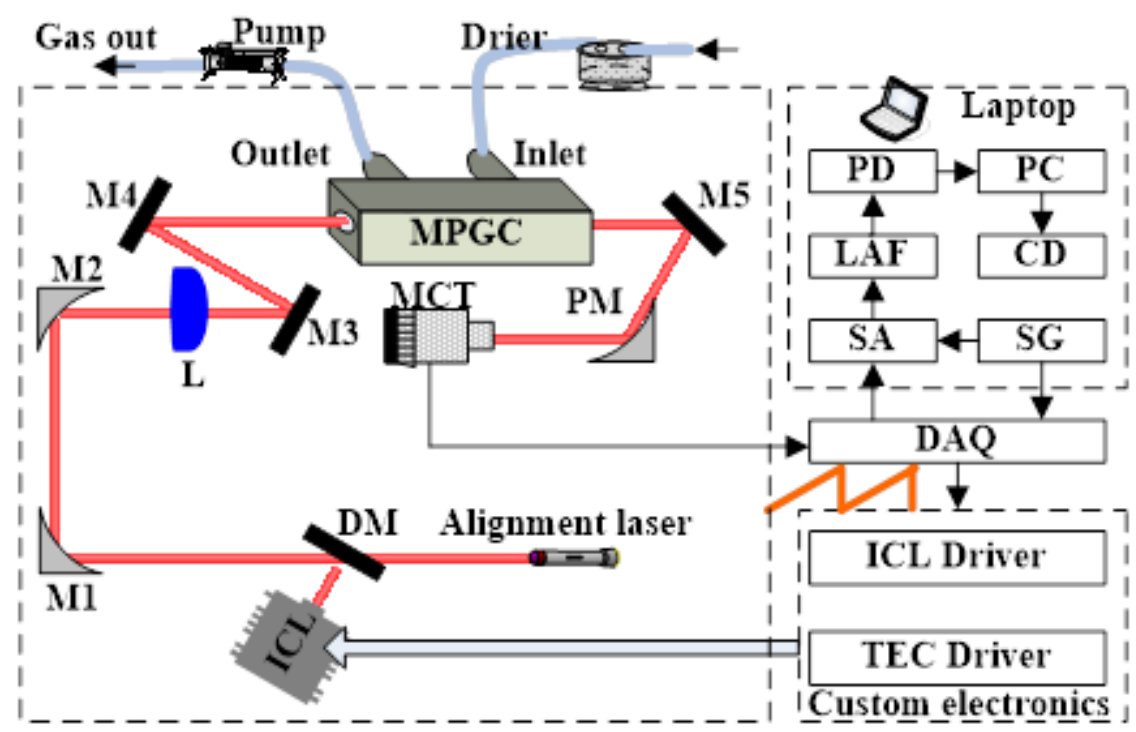

Fig. 1 

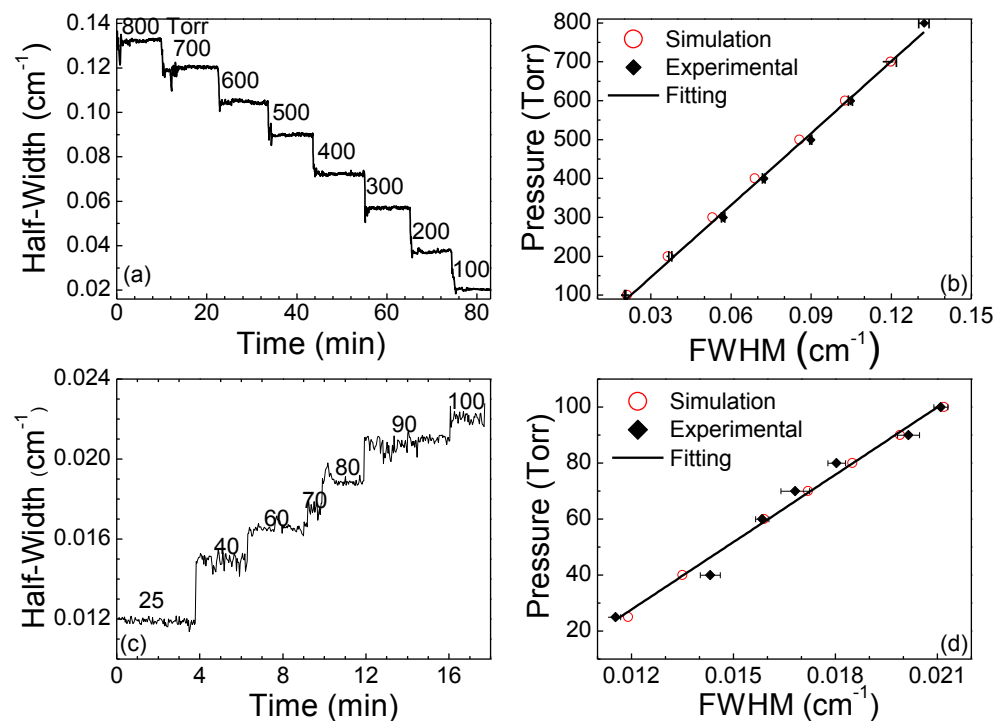

Fig. 2 


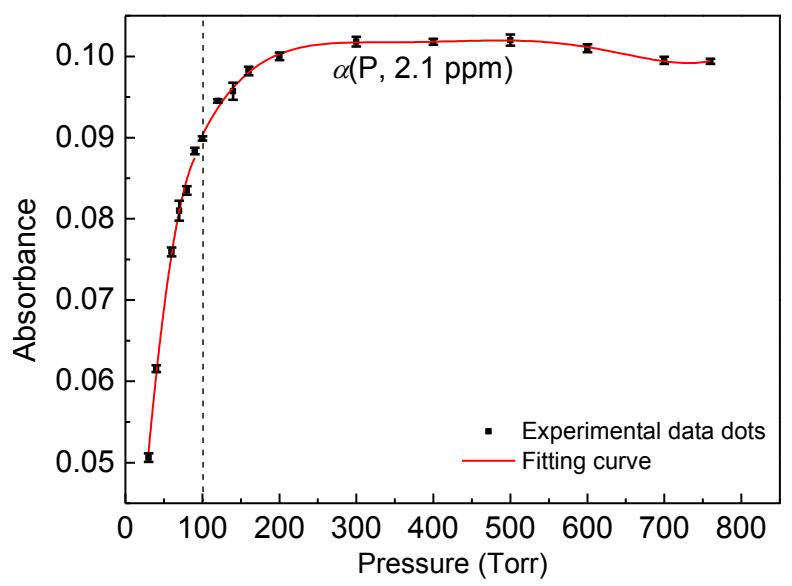

Fig. 3 


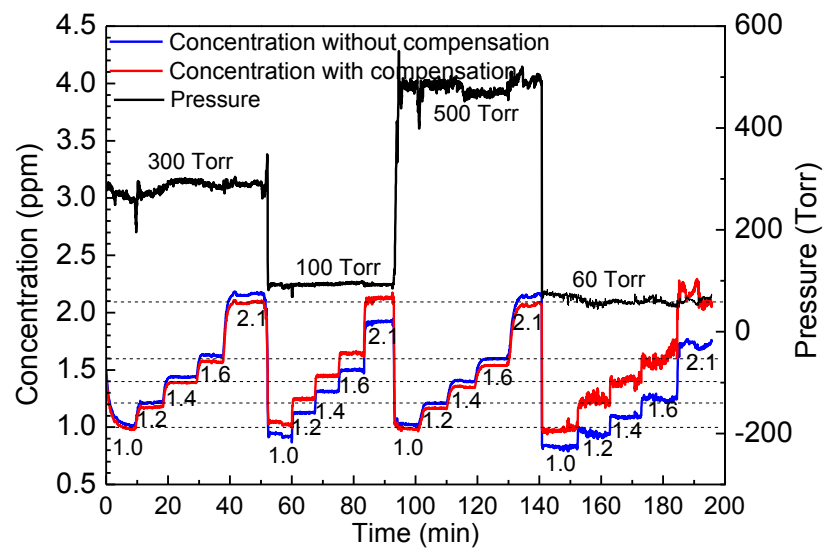

Fig. 4 


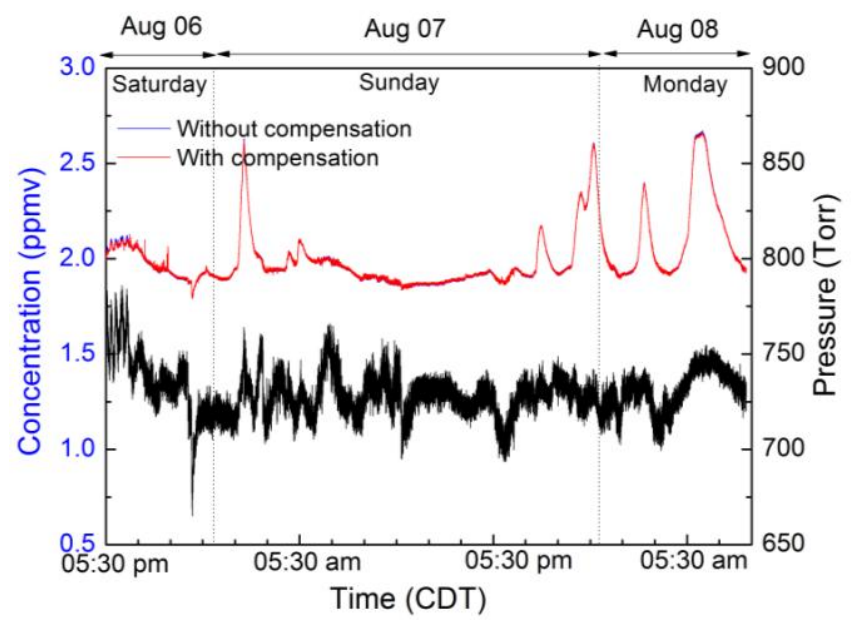

Fig. 5 

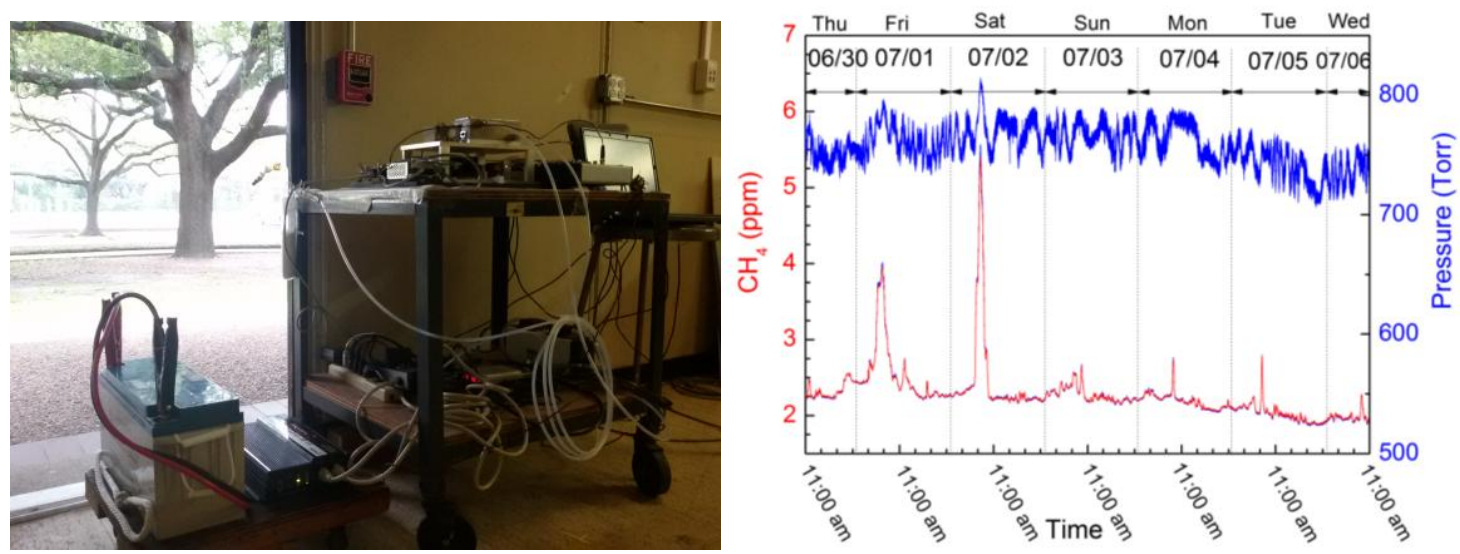

Fig. 6 


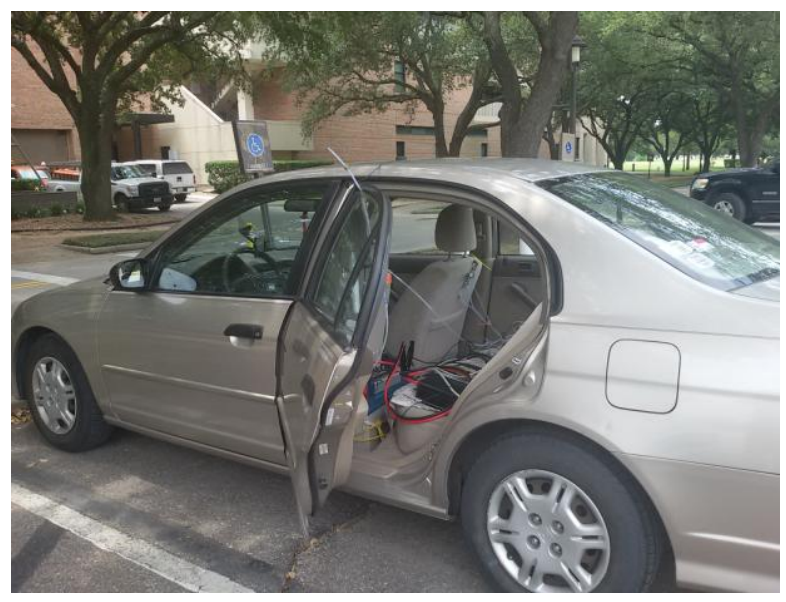

(a)

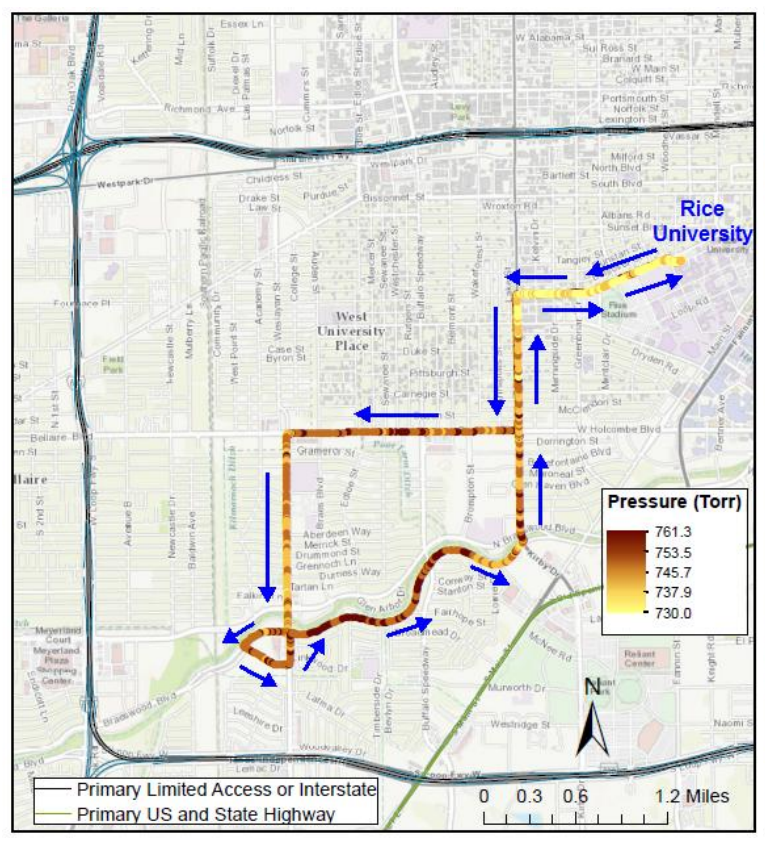

(b)

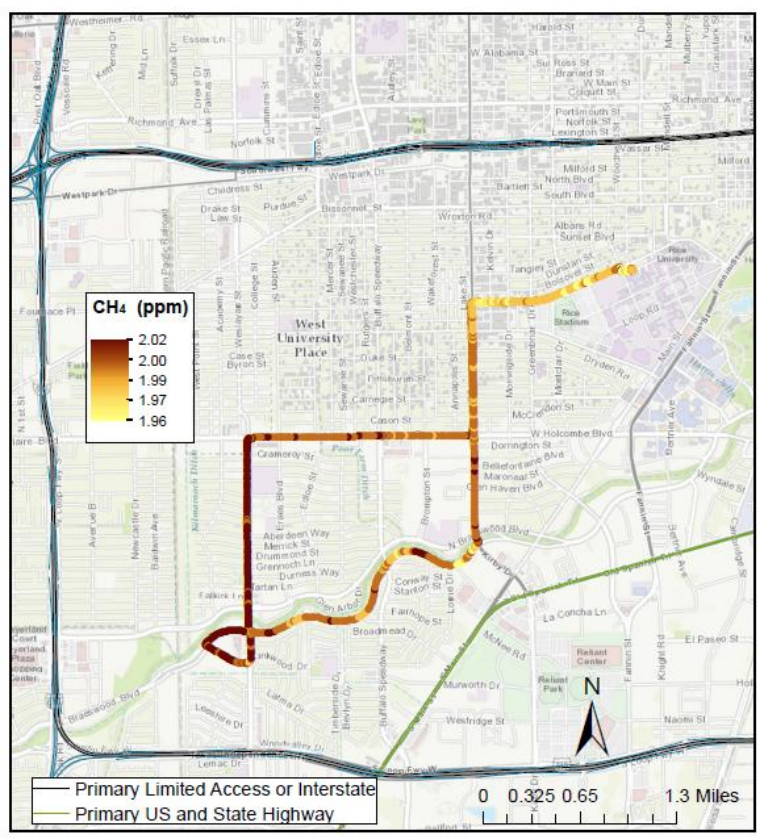

(c)

Fig. 7 